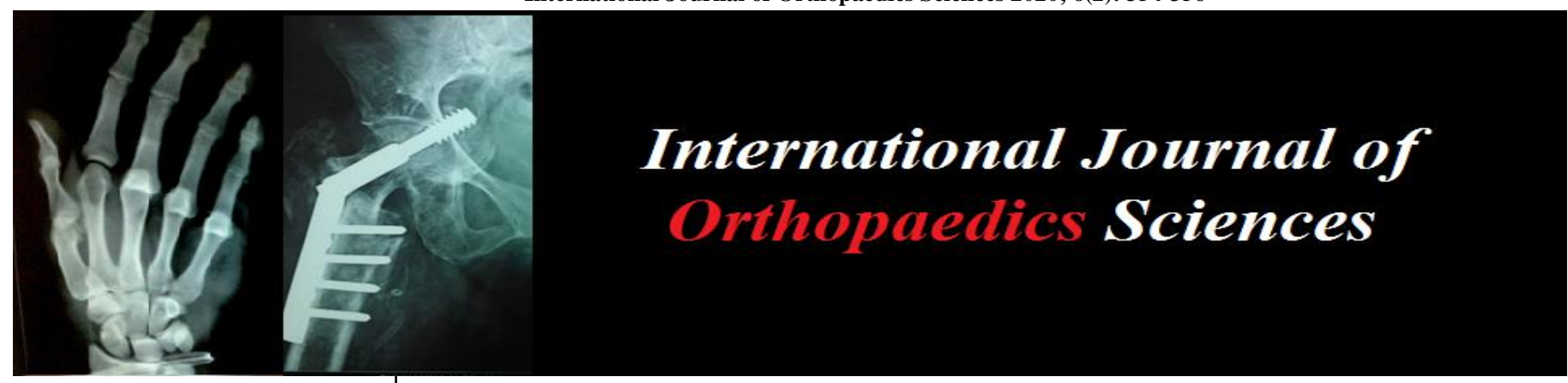

E-ISSN: 2395-1958

P-ISSN: 2706-6630

IJOS 2020; 6(2): 354-356

(C) 2020 IJOS

www.orthopaper.com

Received: 13-01-2020

Accepted: 14-02-2020

Dr. Rajesh K Ambulgekar Prof. \& Head, Department of Orthopedics, Dr. Shankarrao Chavan Govt. Medical College,

Nanded, Maharashtra, India

Dr. Sagar Bhamare

Junior Resident, Department of Orthopedics, Dr. Shankarrao

Chavan Govt. Medical College,

Nanded, Maharashtra, India

Dr. Rohit Somani

Junior Resident, Department of Orthopedics, Dr. Shankarrao

Chavan Govt. Medical College, Nanded, Maharashtra, India
Corresponding Author: Dr. Rajesh K Ambulgekar Prof. \& Head, Department of Orthopedics, Dr. Shankarrao Chavan Govt. Medical College, Nanded, Maharashtra, India

\section{Large enchondroma of metacarpal bone without pathological fracture treated with marginal resection and reconstruction with iliac crest graft: A case report}

\author{
Dr. Rajesh K Ambulgekar, Dr. Sagar Bhamare and Dr. Rohit Somani
}

DOI: https://doi.org/10.22271/ortho.2020.v6.i2f.2067

Abstract

Enchondroma is the benign bone tumor commonly found in phalanx and metacarpal shaft. The most common method of treatment is curettage with or without filling the cavity with bone graft with recurrence rate up to $13 \%$. We chose to present this case of a large enchondroma of fourth metacarpal right hand treated with marginal excision and reconstruction of segmental defect with tricortical iliac crest bone graft with excellent functional and cosmetic result. The reason to report this case was owing to the large size of the tumor without pathological fracture despite of long duration and successful reconstruction of metacarpal with simple technique of autograft.

Keywords: Enchondroma, excision, metacarpal, reconstruction

\section{Introduction}

Enchondroma is common benign bone tumor constituting about $3 \%$ of all bone tumors and about $12 \%-24 \%$ of all benign bone tumors. It is cartilaginous tumor arising from metaphyseodiaphyseal junction, commonly phalanx and metacarpal bones, due to proliferation of remaining hyaline cartilage from the medullary cavity. The common age group is second to fourth decade of life. Many patients are asymptomatic while some present with pain, swelling, cosmetics or pathological fracture. The most common sites are proximal phalanx followed by middle phalanx, metacarpal and distal phalanx in descending order of its frequency. Small and asymptomatic lesion can be managed conservatively with observation with follow-ups while larger painful lesion or lesions with pathological fracture or cosmetic deformity need treatment with curettage and filling the defect with bone graft. Recurrence rate after curettage and bone graft has been reported up to $13 \%$.

Very large lesion with pathological fracture or recurrent lesions are better treated with excision and reconstruction with autograft. We have reported this case of very large enchondroma with extension to subchondral region of right 4th metacarpal shaft treated with excision of the segment and successful reconstruction of metacarpal with simple iliac crest bone graft with excellent result. Informed consent was taken from the patient regarding use of data concerned for publication.

\section{Case Report}

A 31 year female, farmer by occupation, presented with progressive diffuse swelling over dorsum of right hand over 4th metacarpal region. She noticed swelling over dorsum of right hand over 4th metacarpal region 10 years back which was peanut size swelling. It was painless to start with but she experienced mild pain on activity of the hand which increased gradually over time. The swelling increased in size $2 * 1.5 \mathrm{~cm}$ but there's no change in its size since last 5 years. There was no history of trauma or any other constitutional symptoms. On examination, there was ill-defined bony swelling arising from right 4th metacarpal shaft with mild tenderness. There was partial loss of grip due to lack of complete flexion at 4th MCP joint. There was no warmth over the swelling. Sensory and motor function was intact. Plain X ray revealed well defined bony swelling arising from $4^{\text {th }}$ metacarpal shaft extending toc. There was punctate calcification all around the tumor mass. There was no evidence of periosteal reaction. 
FNAC report suggested benign lesion, suggestive of Chondroid tumor. MRI showed features with evidence of a well-defined lytic expansile lesion involving diaphysis of fourth metacarpal in central/medullary location causing paper thinning of the cortex, showing few small calcifications with no pathological fracture or soft tissue extension; lesion suggestive of benign neoplastic etiology, mostly an enchondroma. Hence, Clinicoradiological diagnosis of large enchondroma with extension to subchondral region of the metacarpal shaft without any pathological fracture was made. Though the patient was a working farmer with rigorous activities, she did not have any evidence of pathological fracture which is a rarity in this case. Patient was explained about the disease nature, treatment plan and prognosis. Since tumor was very large with paper thinned cortex, excision of the segment along with lesion with reconstruction of the metacarpal with tricortical iliac crest graft was planned instead of just curettage and bone graft. She was operated under brachial block in supine position with dorsal incision over metacarpal shaft with marginal excision of the tumor, excavation of the cavity was done while isolating and protecting extensor tendons and digital vessels and nerves. The excised segment in block was measured and same length of tricortical iliac crest graft was harvested from the ipsilateral iliac crest. It was used to reconstruct the segmental defect of metacarpal. G-bone (modified hydroxyappatite) was used to further fill the cavity. Haemostasis was achieved and wound was closed with drain insitu and cockup slab given.

Tissue was sent for histopathological examination and was suggestive of lobules of hyaline cartilage encased by a zone of reactive bone with calcification; chondrocytes have nuclei with condensed chromatin. There was no evidence suggestive of malignancy such as chondrosarcoma. Slab was removed in 2 weeks and ROM exercise was started.

There was complete union of the graft at 6 weeks with full ROM and normal grip at 12 weeks. There was no wound complication, pain or evidence of local recurrence till 6 months of follow up. Patient is satisfied with the result and there was no cosmetic problem. Written consent was taken from patient regarding use of these materials for academic publication keeping her identity undisclosed.

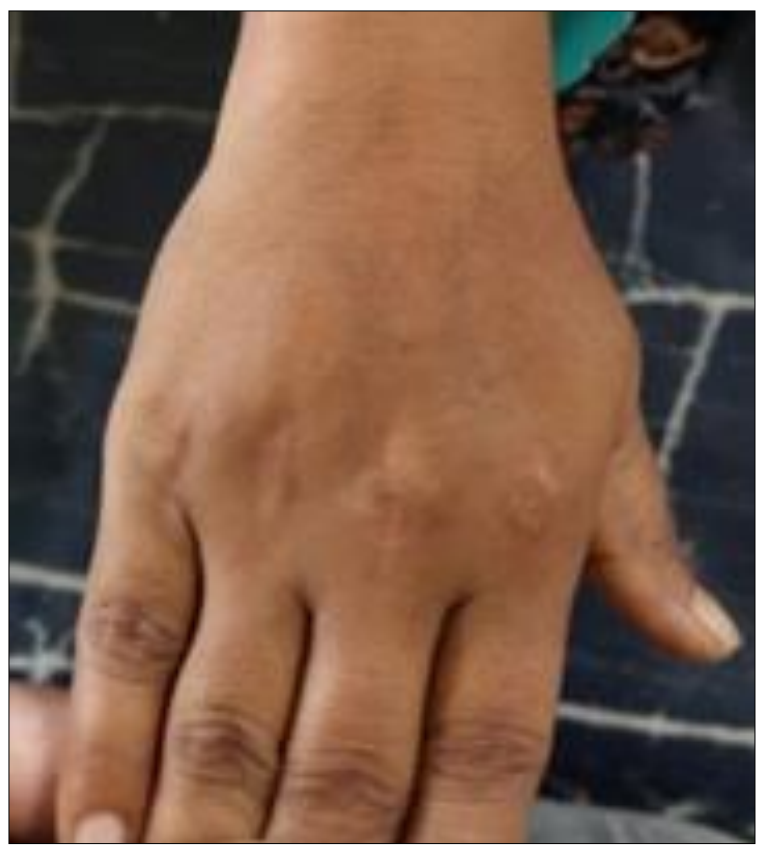

Image 1: Right Hand Clinical photograph showing swelling over right $4^{\text {th }}$ metacarpal region

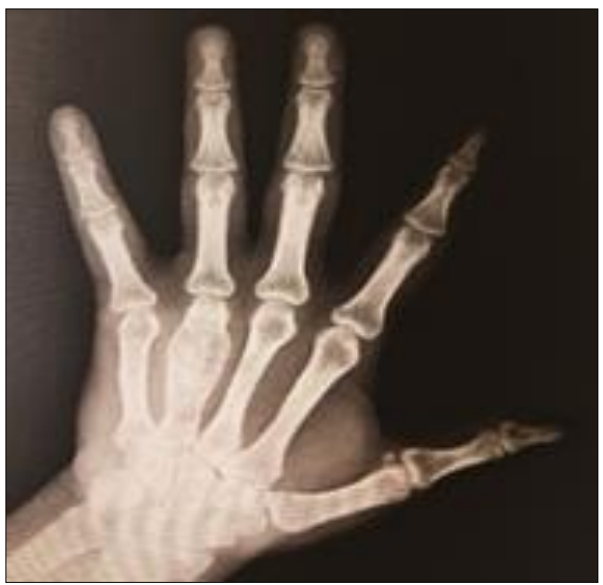

Image 2: X-ray showing well defined bony swelling arising from 4th metacarpal shaft extending to subchondral region of MCP joint.

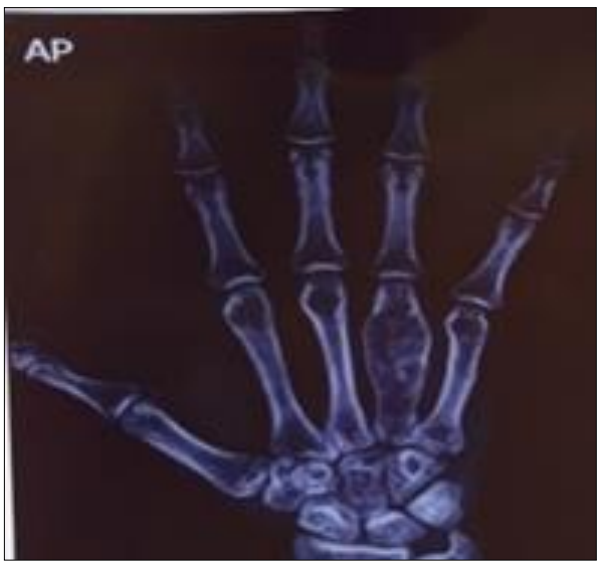

Image 3: MRI showing with evidence of a well-defined lytic expansile lesion involving diaphysis of fourth metacarpal in central/medullary location causing paper thinning of the cortex, showing few small calcifications with no pathological fracture or soft tissue extension

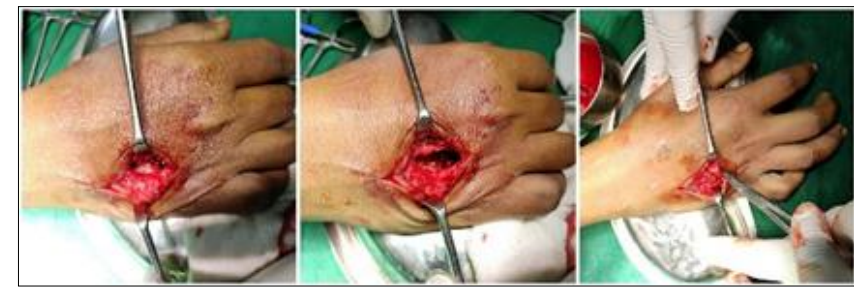

Image 4: (a) Intraoperative enchondroma mass. (b) Excision of mass with segmental defect. (c) tricortical iliac crest graft harvest reconstruction of segmental defect in the metacarpal.

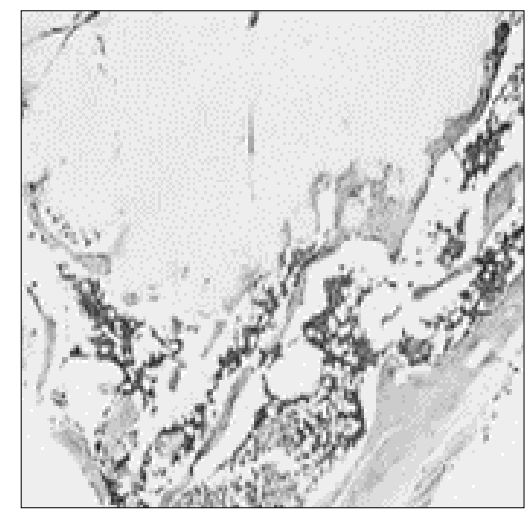

Image 5: histopathological examination and was suggestive of lobules of hyaline cartilage encased by a zone of reactive bone with calcification; chondrocytes have nuclei with condensed chromatin. 


\section{Discussion}

Enchondromas are the second commonest benign bone tumor next to osteochondroma and the most common benign bone tumors in the hand. It is cartilaginous in origin arising from mature hyaline cartilage. It can occur at centre of medullary cavity where it is called enchondroma or can be located eccentrically and is known as periosteal chondroma. The age group varies widely. Hand is the common site for this tumor with prevalence in proximal phalanx, middle phalanx, metacarpal, and distal phalanx in descending order of frequency. Treatment in symptomatic, large tumor or impending pathological fracture is curettage with or without filling the cavity. Small cavity with structurally insignificant enchondroma can be left unfilled and observed in a process to heal itself. But larger cavity needs filling with bone graft while large segmental defect or recurrent lesion needs reconstruction with graft to preserve the function and motion of fingers and hand. In our case, we treated the above mentioned case with marginal excision instead of only curettage as the size was large with papery thin cortex and to avoid chance of recurrence and preserve the function at the same time. There are reports of metacarpal and phalanx reconstruction with cement and hardware after segmental resection but we chose to reconstruct with iliac crest bone graft as it is more natural reconstruction. Reconstruction with bone cement and hardware has drawback of some loss of function of hand with stiffness in many cases. Yalcinka et al has reported successful reconstruction of 5th metacarpal with pathological fracture with structural allograft in recurrent enchondroma treated initially with curettage and bone graft. Sassu et al. has reported a large enchondroma of 3rd metacarpal treated successfully with excision and reconstruction with vascularized fibular graft. Our case has been managed well with simple surgical technique of segmental resection of tumor and reconstruction of the defect with tricortical iliac crest graft resulting in full range of motion with normal grip without any cosmetic issue. The reconstructed graft united and remodeled in such a good manner that it resembled normal metacarpal.

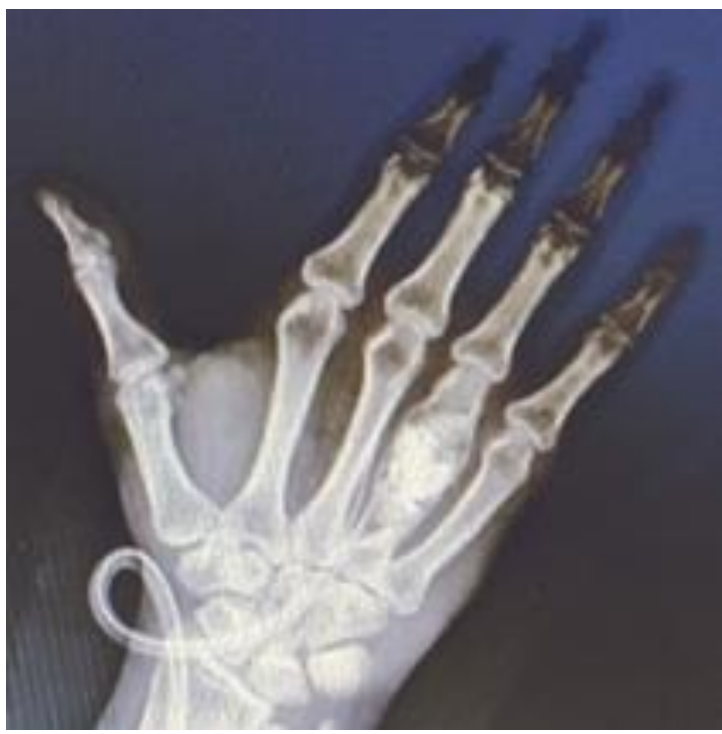

Image 6: Postoperative Xray showing graft filled metacarpal region after mass excision

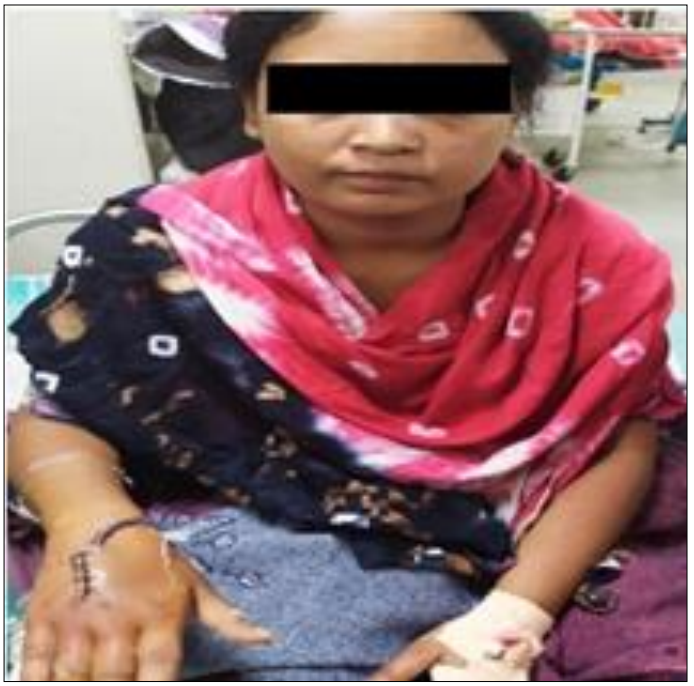

Image 7: Postoperative clinical photograph of the patient with drain insitu.

Conflict of interest: None.

Funding source: None.

\section{References}

1. Yalcinkaya M, Akman YE, Bagatur AE. Recurrent metacarpal enchondroma treated with strut allograft: 14year follow-up. Orthopedics. 2015; 38(7):e647-e650.

2. Sassoon AA, Fitz-Gibbon PD, Harmsen WS, Moran SL. Enchondromas of the hand: Factors affecting recurrence, healing, motion, and malignant transformation. Journal of Hand Surgery. 2012; 37(6):1229-1234.

3. Dorfman HD, Czerniak B. Benign cartilage lesions. In: Dorfman HD, Czerniak B, Bone Tumors. St Louis:Mosby, 1998, 253-276.

4. Kuur E, Hansen SL, Lindequist S. Treatment of solitary enchondromas of the hand. J Hand Surg. 1989; 14B:109112.

5. Sekiya I, Matusi N, Otsuka T, Kobayashi M, Tsuchiya D. The treatment of enchondromas in the hand by endoscopic curettage without bone grafting. J Hand Surg. 1997; 22B:230-234.

6. Shimizu K, Kotoura Y, Nishijima N, Nakamura T. Enchondroma of the distal phalanx of the hand. J Bone Joint Surg. 1997; 79A:898-900.

7. Wulle C. The treatment of enchondroma. J Hand Surg. 1990; 15B:320-330.

8. Bauer RD, Lewis MM, Posner MA. Treatment of enchondroma of the hand with allograft bone. J Hand Surg. 1988; 13A:908-916.

9. Jacob Bickels, James Wittig C, Yehuda Kollender, Kristen Kellar-Graney, Kari Mansour L. Enchondromas of the Hand:Treatment With Curettage and Cemented Internal Fixation. J Hand Surg. 2002; 27A:870-875.

10. Sassu P, Burrai S, Cara L. A case of vascularized fibula bone graft in the treatment of a wide metacarpal enchondroma. Chirurgia. 2013; 26(2):125-128. 